the explanation for the absent lobar perfusion in the other four patients in group 1. However, it is also possible that the bronchial tumour was producing obstructive emphysema, in which case it is postulated that the intra-alveolar pressure may then have been sufficient to prevent the radioactively labelled microemboli from entering the emphysematous zone.

Wagner et al. (1965) reported four cases inf bronchogenic carcinoma in which the scan indicated obstruction of the pulmonary blood flow to a degree unsuspected from the size of the lesion on the chest radiography. These workers inflated balloons in the bronchi of anaesthetized dogs and produced a decrease in pulmonary blood flow, as shown by lung scanning. They postulated that the effect may have resulted from regional hypoxia, but in our group 1 patients the breathing of $100 \%$ oxygen from a facemask before and during the scan produced no alteration in the pattern of perfusion.

In a patient with carcinoma of the bronchus one is not usually concerned with the function of the contralateral lung, provided the overall function of the lungs is reasonable. It was therefore especially interesting to find that in patients in group 2 the perfusion in the contralateral lung was less than that in the lung already partially destroyed by tumour. Thus in almost $20 \%$ of the patients studied it is possible that pneumonectomy would have produced severe respiratory disability or even early postoperative death. Necropsy studies have not as yet been performed on any of these patients, and it can only be conjectured that the "healthy" lung has been previously damaged by thromboembolic or bronchiolar disease.

Patchy areas of decreased perfusion in both lungs in patients presumed to have emphysema, as well as carcinoma of the bronchus (group 3), or in patients with old tuberculous disease (group 4), were not unexpected. Lopez-Majano et al. (1966) described similar changes in emphysema. Nor was it surprising to find areas of decreased perfusion which coincided with the area of radiological abnormality in 21 of the 32 patients in group 5. However, in the remaining 11 patients areas of decreased perfusion were found in radiologically unaffected areas of the same lung, and in five of these there was no evidence of obstruction to lobar or segmental bronchi. As yet we are unable to explain this finding.

\section{Summary}

Lung scans were performed in 50 patients with carcinoma of the bronchus. Unexpected abnormalities of perfusion were found in one-third of them. It is therefore suggested that lung scans should be routine in the preoperative assessment of these patients.

We thank Mrs. Julie Phelps for technical assistance with the scans.

REFERENCES

Ball, W. C., Stewart, P. B., Newsham, L. G. S., and Bates, D. V. (1962) 7. clin. Invest., 41, 519 .

Dyson, N. A., Hugh-Jones, P., Newbery, G. R., Sinclair, J. D., and West, J. B. (1960). Brit. med. Ұ., 1, 231 .

Lopez-Majano, V., Chernick, V., Wagner, H. N., and Dutton, R. E (1964). Radiology, 83, 697.

Lopez-Majano, V., Tow, D. E., and Wagner, H. N. (1966). F. Amer. med. Ass., 197, 81.

Wagner, H. N., Sabiston, D. C., McAfee, J. G., Tow, D., and Stern, H. S. (1964). New Engl. 7. Med., 271, 377.

Wagner, H. N., Lopez-Majano, V., Tow, D. E., and Langan, J. K. (1965). Lancet, 1,344 . West, J. B., Dollery, C. T., and Hugh-Jones, P. (1961). F. clin. Invest.,
40, 1.

\title{
Percutaneous Electrical Cordotomy in Relief of Intractable Pain
}

\author{
S. LIPTON,* M.B., CH.B., F.F.A. R.C.S
}

Anterolateral cordotomy for relief of pain was first performed by Spiller and Martin (1912). It is accepted as the best available method for long-term relief despite its disadvantages. These are: a variable mortality depending on the type of case dealt with, a prolonged period of healing and convalescence in many of the patients with cancer, and a success rate which falls steadily with time (Nathan, 1963a).

The incision into the anterolateral tract is a very small one compared with the extent of the operation, and a number of percutaneous techniques have been described which produce the required lesion without surgery (Mullan et al., 1963, 1965; Rosomoff et al., 1965; Lin et al., 1966).

The percutaneous method has considerable advantages. The mortality is very small and the technique can be used on any patient up to and including the terminal state. If the required level is not attained at the first attempt, or if the level subsequently drops, the procedure is easily repeated. Most patients can be discharged four days after this type of cordotomy.

- Consultant Anaesthetist, Liverpool Regional Neurosurgical Centre, Walton Hospital, Liverpool ; Consultant to Pain Relief Clinic, Liver pool Regional Neurosurgical Centre, Walton Hospital, Liverpool.

\section{Material}

In this unit 52 patients have been treated by percutaneous electrical cordotomy: 44 had intractable pain due to cancer, 4 due to post-herpetic neuralgia, and 4 due to other nonmalignant causes. Ages varied from 31 to 80 years. All patients had been fully investigated before admission -17 were sent by neurosurgeons, 28 were referred by consultants at hospitals throughout the Liverpool region, and 7 were referred by general practitioners. Before the percutaneous cordotomy technique was available this type of patient was treated by surgical cordotomy, by intrathecal phenol, or by a long-acting paravertebral or other block. Often their medical advisers did not seek operative relief. After it was available it has been offered to all patients with intractable pain from cancer. It has also been offered to some patients having non-malignant intractable pain.

Assessment of Pain Relief.- " Relief" means freedom from pain up to the terminal stage of the disease. "Partial relief" means that there is some worth-while benefit obtained from the treatment, such as a statement from the patient to this effect or a reduction in the analgesic drugs needed. Anything not classified as a relief or partial relief is classified as a "failure." 


\section{Technique of Percutaneous Cordotomy}

The method adopted uses a combination of the techniques of Mullan et al. (1965) and Rosomoff et al. (1965). Briefly the Rosomoff radio-frequency current coagulation is used with the Mullan method of delineating the dentate ligament. The apparatus used is mentioned in the Addendum, and a brief description of the technique is as follows.

A spinal needle is inserted laterally between the first and second cervical vertebrae so that the point is anterior to the dentate ligament. An emulsion of cerebrospinal fluid and iophendylate injection B.P. (Ethiodan) is injected. The globules of iophendylate settle on the dentate ligament, and a lateral $x$-ray film shows this as a line (Fig. 1). An electrode is passed down the spinal needle and inserted, just anterior to the dentate ligament, into the anterolateral column of the cord. The depth of penetration of the electrode is estimated from the anteroposterior film, taking the centre line as the midpoint of the odontoid process (Fig. 2).

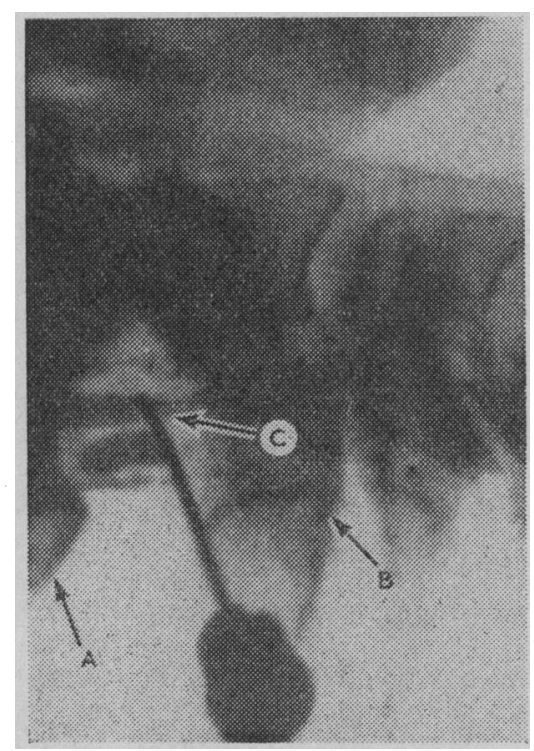

Fig. 1.-Lateral view. A shows the atlas vertebra, $B$ shows the axis vertebra, and $\mathrm{C}$ shows the dentate ligament
by iophendylate emulsion.

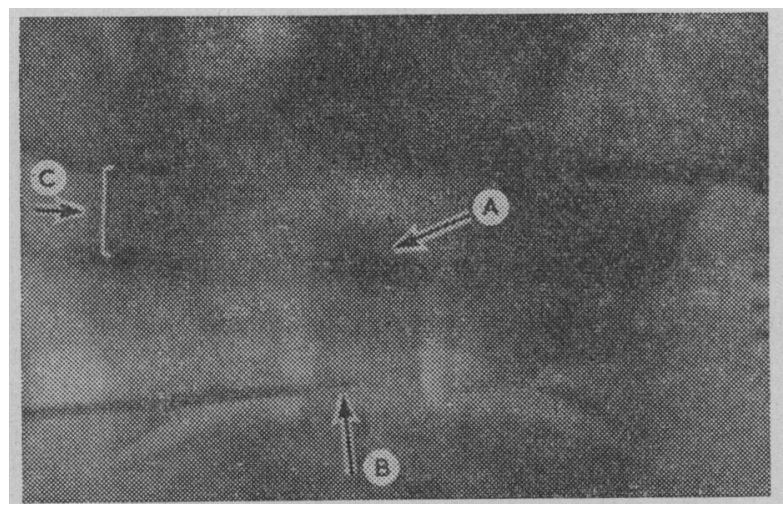

FIG. 2.-Anteroposterior view. A shows the odontoid process, B shows the electrode appearing through the end of the spinal needle, and $C$ shows an artifact caused by the head-holder.

The cord is stimulated by passing a current through the electrode. If the electrode tip is too far posteriorly it is in the motor tract and there will be movement of the ipsilateral body or limbs. If it is too far anteriorly it is in the anterior horn cells and there will be movement of the ipsilateral neck. When it is in the correct position there is no movement and there may be paraesthesiae on the contralateral side. Often there is no sensation at all. When the electrode is in the correct position the anterolateral tract is coagulated and this produces analgesia on the contralateral side. By careful placement of the electrode it is possible to confine the analgesia to a quadrant of the body. Thus analgesia of body and arm or body and leg is possible.

In the early cases Mullan's direct-current technique was used, the analgesia taking 10 minutes to develop. After an initial 10 cases a change to the quicker radio-frequency current was made, and this is the method now in use. A typical case of each technique is described below.

In this series an image intensifier was used in the lateral view and Polaroid $x$-ray film in the anteroposterior view.

\section{Results}

Fifty-two patients have been treated to date, with effective relief of pain in $34(67 \%)$, partial relief in $7(13 \%)$, and 11 failures. They have had 69 cordotomies attempted.

In the 52 patients there were no respiratory or autonomic effects apart from five Horner's syndromes. Headache was invariably present postoperatively, lasting 12 to 48 hours. Hyperalgesia of $\mathrm{C2}$ (ipsilateral side) occurred occasionally. The shortest time for achieving a unilateral cordotomy was 20 minutes, the longest time two and a half hours.

Unilateral Cordotomies.-Forty-four patients had 52 cordotomies attempted, 30 obtaining relief and four partial relief.

Two patients had two attempts each, both failing; they were then treated surgically. Cordotomies on 13 other patients failed at the first attempt and three of these refused further treatment. Of the remaining 10 patients five obtained relief on the second occasion, two died before further treatment could be given, and three are awaiting repeats.

Complications.-Two patients had retention of urine, one of these dying within two days. The other recovered normal function in four days. Two patients developed transient weakness. One recovered completely within 24 hours, the other improved from the fourth day onwards. Analgesia wore off completely in two weeks in two patients and they refused further treatment (these are included in the three refusing treatment mentioned previously). After two months the analgesic area had shrunk enough to uncover pain again in four patients. Two of these had another electrical cordotomy, the result being pain relief in one and partial relief in the other. Two refused. In one patient extension of the malignant process beyond the original analgesic area required treatment. She had a successful repeat electrical cordotomy. One patient developed mild painful dysaesthesiae.

Bilateral Cordotomies.-Eight patients had these, requiring 17 attempts ; five gained relief and three partial relief.

Three patients had three attempts each. Two patients refused further treatment after two attempts each, having obtained a good partial relief. Two patients had bilateral cordotomies done from one side and both were relieved. Three patients were in the terminal state, two being relieved and one having partial relief.

Complications.-Two patients needed continuous bladder drainage after the cordotomy on the second side became effective. One of the terminal cases developed paresis of an arm after the third cordotomy, slow recovery beginning one week later.

\section{Case Histories}

Electrical Cordotomy with Direct Current.-The patient, a woman aged 34, had a sarcomatous spread of the left iliac glands. The entire left leg and groin were grossly oedematous, rigid, and painful. Pain was present from T9 to S5 on the left side. She was sedated with haloperidol $5 \mathrm{mg}$. and phenoperidine $2.5 \mathrm{mg}$. intravenously. She could distinguish pin-prick and co-operated well. 
An electrode was inserted in the right side of the spinal cord as described. Stimulation with $50 \mu \mathrm{A}$ direct current produced no motor response or paraesthesiae. A current of $500 \mu \mathrm{A}$ for four minutes showed a rising level of analgesia from the left knee to the waist on testing with pin-prick. There was no loss of motor function in the limbs, this being tested continuously. $2 \mathrm{~mA}$ direct current for 10 minutes produced analgesia from T1 to S5. The time taken was one and a half hours. There was no disturbance apart from headache the next day. The analgesic level dropped to T3 over the next week and remained at this level until her death six weeks later. She was free from all pain.

Electrical Cordotomy with Radio-frequency Current.-The patient, a man aged 54, had a carcinoma involving the apex of the left lung. He had pain in the left scapula, arm, and hand. He was able to continue with his work as a clerk. Premedication was by haloperidol $5 \mathrm{mg}$. and phenoperidine $1.75 \mathrm{mg}$. A right percutaneous cordotomy was performed as described. The position of the electrode was checked by stimulation, no paraesthesiae being obtained. Coagulation with increasing radio-frequency current up to $80 \mathrm{~mA}$ for 15 seconds produced partial analgesia over the upper left quadrant of the body. The electrode was moved nearer to the dentate ligament. Stimulation produced no paraesthesiae. A radiofrequency current of $40 \mathrm{~mA}$ for 5,10 , and 15 seconds produced analgesia over the upper left quadrant of the body. Further current was applied of $50 \mathrm{~mA}$ for 10,15 , and finally 30 seconds. Analgesia was complete over C4 to T7 and diminished over the rest of the left body. Pain relief was complete. The time taken was 40 minutes. He was discharged home after 48 hours.

\section{Discussion}

The percutaneous electrical cordotomy is remarkably free from side-effects. Rosomoff et al. (1965) emphasize this in their series, but mention a high incidence of Horner's syndrome, which was not a feature of the present series.

Retention of urine is most likely to occur in the bilateral cordotomy, as the two lesions are made at the same level in the spinal cord. It is best to have an interval of a week between them.

As expected, results improve with increasing experience. Pain was relieved in 17 of the last 20 patients treated, and the average time taken to perform the cordotomy was 55 minutes.

There was one patient in this series whose death might have been due to the method. He was a 64-year-old man and had a secondary carcinoma in the left sacroiliac joint, the primary probably being in the lung. Previous to admission he had been treated with intrathecal phenol and alcohol blocks without effect. An effective right percutaneous cordotomy was obtained. He required an indwelling catheter and appeared fit and well with minimal postoperative effects, but collapsed and died 48 hours after the cordotomy. A post-mortem examination did not add any further information.

Though the complication did not occur, mention should be made of an unusual type of respiratory depression that may be found when the descending respiratory fibres in the high cervical cord are bilaterally damaged in operative or electrical cordotomies (Nathan, 1963b ; Hitchcock and Leece, 1967). It may occur with a unilateral cordotomy when there is poor function in the contralateral lung. In this complication the patient can breathe normally while awake, but during sleep the respirations become depressed and need assistance, and the patient may die. Lin et al. (1966) developed an anterior approach to the anterolateral tract to avoid this complication.

\section{Summary}

Fifty-two cases of intractable pain treated by percutaneous electrical cordotomy confirm the simplicity and efficacy of the method. As compared with the operative cordotomy, there is a decreased morbidity and mortality and the convalescence is only a few days. It can be offered to patients in a terminal state. It is more acceptable to patients and their physicians than a major operation. Pain relief was complete in 34 cases and partial relief was obtained in seven.

\section{Addendum}

The lesion generator and stimulator was designed and made by Mr. John Blackett, of Messrs. Matburn \& Co. Ltd., 20 Emerald Street, London W.C.1.

The electrodes used were 0.01 or 0.015 -in. ( 0.25 to $0.38 \mathrm{~mm}$.) diameter, $30 \% / 70 \%$ iridium/platinum alloy.

The epoxy resin used for insulation was provided by $\mathrm{Mr}$. P. A. Letts, Manager, Kollercast Division, Beck Koller \& Co., 103 High Street, Thame, Oxon.

The Polaroid film (57/3000) and special cassette XR 7 was supplied by Messrs. Polaroid (U.K.) Ltd., Rosanne House, Welwyn Garden City, Herts.

The head holder and restrainer was designed by Mr. S. Cope, Superintendent Radiographer, Walton Hospital, Liverpool.

This work was supported by a grant from the Liverpool Regional Hospital Board.

I wish to thank the staff of the Neurosurgical Centre and the $X$-ray Department, Walton Hospital, for their help in getting this project started. In particular I wish to thank Mr. A. Sutcliffe Kerr for his helpful discussions and encouragement and Dr. J. V. Occleshaw for his aid in reading the films.

\section{REFERENCES}

Hitchcock, E., and Leece, B. (1967). f. Neurosurg., 27, 320. Lin, P. M., Gildenberg, P. L., and Polakoff, P. P. (1966). F. Neurosurg., $25,553$.

Mulian, S., Harper, P. V., Hekmatpanah, J., Torres, H., and Dobbin, G. (1963). F. Neurosurg., 20, 931.

Mullan, S., Hekmatpanah, J., Dobben, G., and Beckman, F. (1965). 7. Neurosurg., 22, 548 .

Nathan, P. W. (19632). 尹. Neurol. Neurosurg. Psychiat., 26, 353.

Rosomoff, H. L., Carroll, F., Brown, J., and Sheptak, P. (1965). 3. Neurosurg., 23, 639.

Spiller, W. G., and Martin, E. (1912). J. Amer. med. Ass., 58, 1489. 